\title{
A Midcourse Guidance Law for Missiles with Thrust Vector Control
}

\author{
Fu-Kuang Yeh ${ }^{1}$, Hsiuan-Hau Chien ${ }^{1}$, and Li-Chen Fu ${ }^{1,2}$ \\ Department of Electrical Engineering ${ }^{1}$ \\ Department of Computer Science and Information Engineering ${ }^{2}$ \\ National Taiwan University, Taipei, Taiwan, R.O.C \\ Email: lichen@ccms.ntu.edu.tw
}

Keywords: Sliding Mode, Optimal Control, Target Tracking, Nonlinear System, Quaternion

\begin{abstract}
A nonlinear missile guidance controller with Thrust Vector Control (TVC) inputs for the interception of a theater ballistic missile is presented. In this paper, the midcourse phase of the missile is investigated, during which many non-ideal conditions must be considered, such as variation of the inertia of the missile and existence of the aerodynamic force, and an effect controller is designed take into account the properties of TVC.

Specifically, a 3 DOF optimal midcourse guidance law is designed to minimize the control effort and the distance between the missile and the target. The asymptotic stability of the overall system is proved by Lyapunov theory. Extensive simulations are conducted to validate the effectiveness of the proposed guidance law for TVC.
\end{abstract}

\section{Nomenclature}

$\begin{array}{ll}\vec{a} & \text { Acceleration vector } \\ \vec{d} & \text { Disturbances } \\ d_{p} & \text { Pitch angle of propellant } \\ d_{y} & \text { Yaw angle of propellant } \\ \vec{F} & \text { Thrust vector } \\ \vec{g} & \text { Gravitational acceleration vector } \\ J & \text { Moment of inertial matrix } \\ J_{0} & \text { Nominal parts of } J\end{array}$

0-7803-6495-3/01/\$10.00 @ 2001 AACC

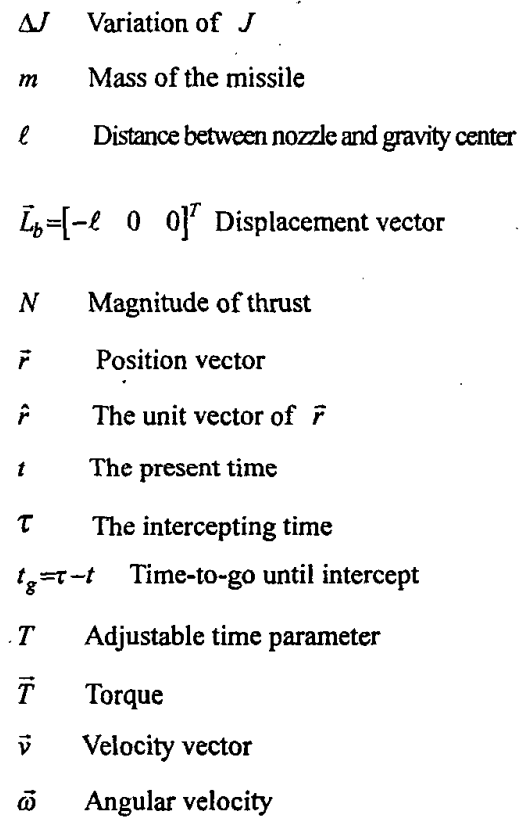

\section{Subscripts}

$b$ The body coordinate frame

d Desired

$i \quad$ The inertial coordinate frame

$M \quad$ Missile

$p \quad$ Perpendicular to LOS

$T \quad$ Target

\section{Introduction}

Based on the concept of the PN guidance law, the constant bearing guidance is often employed on the Bank-to-Turn (BTT) missiles [1,2], whereas a different kind of guidance law, namely, zero-sliding 
guidance law aims at eliminating the sliding velocity between the missile and the target in the direction normal to LOS [3]. Ha and Chong derived a new command to line-of-sight (CLOS) guidance law for short-range surface to air missile via feedback linearization [4] and its modified version [5] with improved performance.

In order to utilize the prior information on the future target maneuvers or on the autopilot lags, optimal guidance law based on the optimal control theory [6] has been investigated after 1960's, although that guidance law requires more measurements than the PN guidance law $[8,9,10]$. A new optimal guidance law without the estimation of the interception time is proposed to deal with situation where the accurate time-to-go is unavailable [11].

On the other hand, attitude control is another important issue to be addressed for successful missile operation. It is quite often that quaternion representation has been adopted to describe the attitude of a spacecraft $[12,13]$, because it is recognized as a kind of attitude global representation. To account for with the non-ideal factors of the spacecraft under attitude control, the sliding mode control has been employed by Chen [15], whereas the adaptive control has been employed by Slotine[16]

\section{Equations of Motion}

The motion of a missile can be described in two parts as follows

$$
\begin{array}{ll}
\text { Translation: } & \dot{\vec{v}}_{M}=\vec{a}_{M}+\vec{g}_{M}, \dot{\vec{r}}_{M}=\dot{\vec{v}}_{M} \\
\text { Rotating: } & J \dot{\vec{\omega}}=-\vec{\omega} \times(J \vec{\omega})+\vec{T}
\end{array}
$$

Referring to Fig. 1, the force and torque exerted on the missile can be expressed as

$$
\vec{F}_{b}=N\left[\begin{array}{l}
\cos d_{y} \cos d_{p} \\
\cos d_{p} \sin d_{y} \\
\sin d_{p}
\end{array}\right]
$$

and

$$
\vec{T}_{b}=I N\left[\begin{array}{l}
0 \\
\sin d_{p} \\
-\cos d_{y} \sin d_{p}
\end{array}\right]
$$

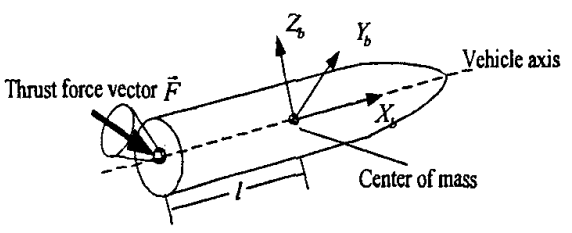

Figure 1: TVC Actuator with Single Nozzle

One can accomplish the transformation of vectors from the inertial coordinate system to the body coordinate system as follows

$$
\vec{F}_{i}=B_{b} \vec{F}_{b}
$$

From eqs.(1) to (5), the dynamic equations of the missile can then be formulated as

$$
\begin{gathered}
\dot{\vec{v}}_{M}=\vec{F}_{i} / m+\vec{g}_{M}=\left(B_{b} \vec{F}_{b}\right) / m+\vec{g}_{M} \\
J \dot{\bar{\omega}}=-\vec{\omega} \times(J \vec{\omega})+l N\left[\begin{array}{l}
0 \\
\sin d_{p} \\
-\cos d_{y} \sin d_{p}
\end{array}\right]
\end{gathered}
$$

\section{Guidance System Design}

A modified optimal guidance law without estimation of time-to-go is designed by not considering the relative motion along the line of sight. The equations of relative motion perpendicular to the LOS are as follows

$$
\dot{\vec{v}}_{p}(t)=-\vec{a}_{M p}(t), \dot{\vec{r}}_{p}(t)=\vec{v}_{p}(t)
$$

The proposed optiamal guidnce law is then given as

$$
\vec{a}_{M p}(t)=\left[\begin{array}{ll}
3 / T^{2} & 3 / T
\end{array}\right]\left[\begin{array}{c}
\vec{r}_{p}(t) \\
\vec{v}_{p}(t)
\end{array}\right] .
$$

Substituting (9) into (8), we get a new state equation without input

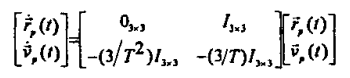


Therefore, by [7] we know that for any positive definite matrix $Q$ we can always find a matrix $\bar{P}$ such that $\bar{A}^{T} \bar{P}+\bar{P} \bar{A}=-Q$ and $\bar{P}$ is a positive definite matrix. Thus, we define a Lyapunov function $V_{G}=\left[\begin{array}{ll}\vec{r}_{p} & \vec{v}_{p}^{T}\end{array}\right] \vec{P}\left[\begin{array}{l}\vec{r}_{p} \\ \vec{v}_{p}\end{array}\right]$, whose time derivative of the Lyapunov function is derived as

$$
\dot{V}_{G}=-\left[\begin{array}{ll}
\vec{r}_{p}^{T} & \vec{v}_{p}{ }^{T}
\end{array}\right] Q\left[\begin{array}{l}
\vec{r}_{p} \\
\vec{v}_{p}
\end{array}\right] \leq 0 .
$$

If we define $Q=I_{6 \times 6}$, then we can get the matrix $\bar{P}$ with the following form as follows

$$
\bar{P}=\left[\begin{array}{cc}
\left(\frac{2}{3} T+\frac{1}{2 T}\right) I_{3 \times 3} & \frac{T^{2}}{6} I_{3 \times 3} \\
\frac{T^{2}}{6} I_{3 \times 3} & \left(\frac{T^{3}}{18}+\frac{T}{6}\right) I_{3 \times 3}
\end{array}\right],
$$

which can be verified to be a positive definite matrix by checking its eigenvalues. Therefore, the system (10) can be shown exponentially stable.

\section{Autopilot System Design}

From Eq.(2) and quaternion, the mathematical model of a missile can be derived as

$$
\begin{aligned}
& \dot{q}_{e}=\frac{1}{2}\left\langle\vec{q}_{e} \times\right\rangle \vec{\omega}_{e}+\frac{1}{2} q_{e 4} \vec{\omega}_{e} \\
& \dot{q}_{e 4}=-\frac{1}{2} \vec{\omega}_{e}^{T} \bar{q}_{e} \\
& J \dot{\hat{\omega}}=-\vec{\omega} \times(J \vec{\omega})+\vec{T}+\vec{d}
\end{aligned}
$$

where $\vec{\omega}_{e}=\vec{\omega}-\vec{\omega}_{d}$ is the error between angular velocities at the present attitude and the desired attitude, respectively.

Let us choose the sliding manifold as

$$
S_{\alpha}=P \vec{q}_{e}+\vec{\omega}_{e}
$$

where $P=\operatorname{diag}\left[\begin{array}{lll}p_{1} & p_{2} & p_{3}\end{array}\right]$ is a positive definite diagonal matrix. According to the derivations by $\mathrm{Fu}$ et al. [3], the system origin $\left(\vec{q}_{e}, \vec{\omega}_{e}\right)=\left(0_{3 \times 1}, 0_{3 \times 1}\right)$ can be shown to be exponentially stable.

From the fact that $J$ is symmetric and positive definite, the candidate of Lyapunov function is set as

$$
V_{s}=\frac{1}{2} S_{a}^{T} J S_{a}
$$

Taking the first derivative of $V_{s}$, we have

$$
\begin{aligned}
\dot{V}_{s} & =S_{a}^{T}\left[-\vec{\omega} \times(J \vec{\omega})+\vec{T}+\vec{d}-J \dot{\vec{\omega}}_{d}\right. \\
& \left.+J P\left(\frac{1}{2}\left\langle\bar{q}_{e} \times\right) \vec{\omega}_{e}+\frac{1}{2} q_{e} d \vec{\omega}_{e}\right)\right]
\end{aligned}
$$

Let the control law be

$$
\begin{aligned}
\vec{T} & =-J_{0} P\left(\frac{1}{2}\left\langle\bar{q}_{e} \times\right) \vec{\omega}_{e}+\frac{1}{2} q_{e 4} \vec{\omega}_{e}\right) \\
& +\vec{\omega} \times\left(J_{0} \vec{\omega}\right)+J_{0} \dot{\omega}_{d}+\vec{\tau}
\end{aligned}
$$

where $\vec{\tau}^{\prime}=\left[\begin{array}{lll}\tau_{1}^{\prime} & \tau_{2}^{\prime} & \tau_{3}^{\prime}\end{array}\right]^{T}, \tau_{i}^{\prime}=-k_{i} \cdot \operatorname{sgn}\left(S_{a i}\right), i=1,2,3$

$\operatorname{sgn}(\cdot)$ is a sign function, then (18) becomes

$$
\dot{V}_{s}=\sum_{i=1}^{3}-k_{i} \mid S_{a i}\left[1-\frac{\delta_{i}}{k_{i}} \operatorname{sgn}\left(S_{a i}\right)\right]
$$

where

$$
\vec{\delta}=\vec{\omega} \times(\Delta J \vec{\omega})+\vec{d}+\Delta J \dot{\vec{\omega}}_{d}+\Delta J P\left(\frac{1}{2}\left\langle\bar{q}_{e} \times\right\rangle \vec{\omega}_{e}+\frac{1}{2} q_{e 4} \vec{\omega}_{e}\right) .
$$

Assume the external disturbances $\vec{d}$ and uncertain parameters $\Delta J$ are all bounded, then the upper bound of $\left|\delta_{i}\right|$ can be found and denoted as $\delta_{i}^{\max }$. It is evident that if we choose $k_{i}=\delta_{i}^{\max }$ for $i=1,2,3$, then (20) becomes

$$
\dot{V}_{s}=-\sum_{i=1}^{3} \delta_{i}^{\max } \mid S_{a i}\left[1-\frac{\delta_{i}}{\delta_{i}^{\max }} \operatorname{sgn}\left(S_{a j}\right)\right]<0
$$

for $S_{a} \neq 0$. Therefore, the reaching and sliding of the sliding mode $S_{a}=0$ is guaranteed.

Generally, the stability of an integrated system cannot be guaranteed by the stability of each individual subsystem of the integrated system, and thus the closed-loop stability of the overall system must be re-evaluated. In other words, if the desired acceleration is $\vec{a}_{M p}+\vec{r}^{\prime}$, the actual acceleration applied on the missile is

$$
\vec{a}_{M}=B_{b} B^{T}\left(q_{c}\right) B_{b}^{T}\left(\vec{a}_{M p}+\vec{r}^{T}\right)
$$


where $B\left(q_{e}\right)=I_{3 \times 3}+2\left(\bar{q}_{e} \times\right)\left(\bar{q}_{e} x\right)+2 q_{e 4}\left(\bar{q}_{e} x\right)$, and

$\vec{r}^{\prime}=\sqrt{(N / m)^{2}-\left|\vec{a}_{M p}\right|^{2}} \hat{r}$, then we get a new state

equation as follows

$$
\begin{aligned}
& {\left[\begin{array}{c}
\dot{\vec{r}}_{p} \\
\dot{\vec{v}}_{p}
\end{array}\right]=\left[\begin{array}{ll}
0_{3 \times 3} & I_{3 \times 3} \\
0_{3 \times 3} & 0_{3 \times 3}
\end{array}\right]\left[\begin{array}{l}
\vec{r}_{p} \\
\vec{v}_{p}
\end{array}\right]}
\end{aligned}
$$

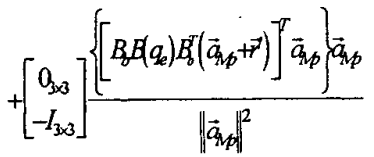

Then, the time derivative of the Lyapunov function can be derived as

$$
\begin{gathered}
\dot{V}_{G}=-\left[\begin{array}{ll}
\vec{r}_{p}^{T} & \left.\vec{v}_{p}^{T}\right] d Q_{[}^{\vec{r}_{p}} \\
\vec{v}_{p}
\end{array}\right]-2\left(\left[\begin{array}{ll}
\vec{r}_{p}^{T} & \vec{v}_{p}^{T}
\end{array}\right]\left[\begin{array}{l}
\vec{r}_{p} \\
\vec{v}_{p}
\end{array}\right]\right) \cdot \\
\frac{\left\{\left[\bar{B}\left(q_{e}\right) \vec{F}_{b}\right]^{T} \vec{a}_{M p}^{\prime \prime}\right\}}{\left\|\vec{a}_{M p}\right\|^{2}}
\end{gathered}
$$

where $\vec{F}_{b}=B_{b}^{T}\left(\vec{a}_{\Lambda \phi}+\vec{r}\right)$, and $\vec{a}_{M p}^{\prime \prime}=B_{b}^{r} \vec{a}_{M p}, \bar{Q}$ is a matrix of the form as follows

$$
\begin{gathered}
\bar{Q}=\bar{P}\left[\begin{array}{cc}
0_{3 \times 3} & 0_{3 \times 3} \\
-3 / T^{2} \cdot I_{3 \times 3} & -3 / T \cdot I_{3 \times 3}
\end{array}\right]+ \\
{\left[\begin{array}{cc}
0_{3 \times 3} & -3 / T^{2} \cdot I_{3 \times 3} \\
0_{3 \times 3} & -3 / T \cdot I_{3 \times 3}
\end{array}\right] \bar{P}}
\end{gathered}
$$

Note that we use the fact that $\vec{r}^{T} \vec{a}_{M p}=0$, and

$\bar{B}\left(q_{e}\right)=\left\langle\bar{q}_{e} \times\right\rangle\left\langle\bar{q}_{e} \times\right\rangle+q_{e 4}\left\langle\bar{q}_{e} \times\right\rangle$.

We define the Lyapunov function candidate of the overall system as

$$
V=\frac{1}{2} S_{a}^{T} J S_{a}+V_{G}
$$

The time derivative of the Lyapunov function can be derived as

$$
\begin{aligned}
\dot{V}= & S_{a}^{T}\left[-\vec{\omega} \times(J \vec{\omega})+\vec{T}+\vec{d}-J \dot{\omega}_{d}+J \frac{p}{2}\left(\left\langle\left(\bar{q}_{e} \times\right) \vec{\omega}_{e}\right.\right.\right. \\
& \left.\left.+q_{e 4} \vec{\omega}_{e}\right)+\frac{K_{1}}{p}\left(\vec{F}_{b} \times\right)\left(\left\langle\vec{q}_{e} \times\right\rangle+q_{e 4} I_{3 \times 3}\right) \vec{a}_{M p}^{\prime \prime}\right] \\
& -\frac{K_{1}}{p} \vec{a}_{M p}^{\prime \prime}\left(\left\langle\vec{q}_{e} \times\right)+q_{e 4} I_{3 \times 3}\right)\left\langle\vec{F}_{b} \times\right\rangle \vec{\omega}_{e}
\end{aligned}
$$

$$
-\left[\begin{array}{ll}
\vec{r}_{p}^{T} & \vec{v}_{p}^{T}
\end{array}\right] Q\left[\begin{array}{l}
\vec{r}_{p} \\
\vec{v}_{p}
\end{array}\right]
$$

To guarantee the stability of the overall system, the following assumption is introduced to specify the requirement of the adjustable parameters:

Assumption Through the entire course of midcourse phase, we can always find appropriate gain $p$ and convergence time such that

$$
\frac{\left(2 \vec{a}_{M p}^{\prime \prime}\left(\left\langle\bar{q}_{e} \times\right\rangle+q_{e 4} I_{3 \times 3}\right)\left(\vec{F}_{b} \times\right\rangle \vec{\omega}_{e}\right)}{p} \vec{Q}<Q
$$

By the assumption, (27) can be written as

$$
\begin{aligned}
& \dot{V}=S_{a}^{Y}\left[-\vec{\omega} \times(J \vec{\omega})+\vec{T}+\vec{d}-J \dot{\omega}_{d}+J \frac{p}{2}\left(\left\langle\bar{q}_{e} \times\right) \vec{\omega}_{e}\right.\right. \\
& \left.\left.+q_{e 4} \vec{\omega}_{e}\right)+\frac{K_{1}}{p}\left\langle\vec{F}_{b} \times\right\rangle\left(\left(\vec{q}_{e} \times\right\rangle+q_{e 4} I_{3 \times 3}\right) \vec{a}_{M p}^{T}\right] \\
& -\left[\begin{array}{ll}
\vec{r}_{p}^{T} & \vec{v}_{p}^{T}
\end{array}\right] Q^{\prime}\left[\begin{array}{c}
\vec{r}_{p} \\
\vec{v}_{p}
\end{array}\right],
\end{aligned}
$$

where $Q=Q-\frac{\left(2 \vec{a}_{M p}^{*}\left(\left\langle\bar{q}_{e} \times\right\rangle+q_{e 4} I_{3 \times 3}\right)\left\langle\bar{F}_{b} \times\right\rangle \vec{\omega}_{e}\right)}{p} \bar{Q}$ is a positive definite matrix.

$$
\text { The Lyapunov function } V_{s}=\frac{1}{2} S_{a}^{T} J S_{a} \text { is used }
$$

to check the stability of the autopilot system. Then,

(29) can be written as

$$
\dot{V}=S_{a}^{T}\left[\begin{array}{ll}
\vec{\delta}_{1}+\vec{\tau}^{\prime}
\end{array}\right]-\left[\begin{array}{ll}
\vec{r}_{p}^{T} & \vec{v}_{p}^{T}
\end{array}\right] Q^{\prime}\left[\begin{array}{l}
\vec{r}_{p} \\
\vec{v}_{p}
\end{array}\right],
$$

and $\quad \dot{V}_{s}=S_{a}^{T}\left[\vec{\delta}_{2}+\vec{\tau}\right]$, where

$$
\begin{aligned}
& \vec{\delta}_{1}=\left[\begin{array}{lll}
\delta_{11} & \delta_{12} & \delta_{13}
\end{array}\right]^{T} \\
& =\vec{\omega} \times(\Delta J \vec{\omega})+\vec{d}+\Delta / \dot{\vec{\omega}}_{d} \\
& +p \Delta J\left(\frac{1}{2}\left\langle\bar{q}_{e} \times\right\rangle \vec{\omega}_{e}+\frac{1}{2} q_{e 4} \vec{\omega}_{e}\right) \\
& -\frac{K_{1}}{p} \vec{a}_{M p}^{* T}\left(\left\langle\bar{q}_{e} \times\right\rangle-q_{e 4} I_{3 \times 3}\right)\left\langle\vec{F}_{b} \times\right\rangle \vec{\omega}_{e}, \\
& \text { and } \vec{\delta}_{2}=\left[\begin{array}{lll}
\delta_{21} & \delta_{22} & \delta_{23}
\end{array}\right]^{T}
\end{aligned}
$$




$$
\begin{aligned}
& =\vec{\omega} \times(\Delta J \vec{\omega})+\bar{d}+\Delta / \dot{\bar{\omega}}_{d} \\
& +p \Delta\left(\frac{1}{2}\left\langle\bar{q}_{e} \times\right\rangle \vec{\omega}_{e}+\frac{1}{2} q_{e 4} \vec{\omega}_{e}\right)
\end{aligned}
$$

Thus the upper bound of $\left|\delta_{I i}\right|$ and $\left|\delta_{2 i}\right|$ can be found and we define $\delta_{i}^{\max }=\max \left(\left|\delta_{1 i}\right|,\left|\delta_{2 i}\right|\right)$. Then, (30)

becomes

$$
\begin{aligned}
& \dot{V}=-\sum_{i=1}^{3} \delta_{i}^{\max }\left|S_{a i}\right|\left[1-\frac{\delta_{1 i}}{\delta_{i}^{\max }} \operatorname{sgn}\left(S_{a i}\right)\right] \\
& -\left[\begin{array}{ll}
\vec{r}_{p}{ }^{T} & \vec{v}_{p}{ }^{r}
\end{array}\right] Q^{\prime}\left[\begin{array}{l}
\vec{r}_{p} \\
\vec{v}_{p}
\end{array}\right]<0
\end{aligned}
$$

and

$$
\dot{V}_{s}=-\sum_{i=1}^{3} \delta_{i}^{\max } \mid S_{a i}\left[1 \frac{\delta_{2 i}}{\delta_{i}^{\max }} \operatorname{sgn}\left(S_{a i}\right)\right]<0
$$

According to Lyapunov stability theory, the stability of the overall system and the autopilot system is proved at the same time.

\section{Simulations}

The validity of the guidance and the autopilot systems of the integrated midcourse systems proposed in $\sec .3$ and $\sec .4$ will be verified through various simulations in this section. Here, we consider the variation of the inertia of the missile and the disturbance resulting from the aerodynamic force. Thus, the inertia matrix we use here is as follows

$$
J=J_{0}+\Delta J\left(\mathrm{~kg} \cdot \mathrm{m}^{2}\right)
$$

where

$$
\begin{aligned}
& J_{0}=\left[\begin{array}{ccc}
867 & 0 & 0 \\
0 & 1926.3 & 0 \\
0 & 0 & 1926.3
\end{array}\right] \\
& \left|\Delta J_{11}\right|<693.6\left(80 \% \text { of } J_{11}\right) \\
& \left|\Delta J_{22}\right|<1541\left(80 \% \text { of } J_{22}\right) \\
& \left|\Delta J_{33}\right|<1541\left(80 \% \text { of } J_{33}\right),
\end{aligned}
$$

Finally, we use the ending condition in the midcourse as the initial condition for the subsequent terminal guidance and control, and then check whether the final intercept due to Fu et al. [3] may be successful. Thus the midcourse phase offer applicable ending conditions to ensure the interception of the missile. And the remarkable success combining the midcourse and terminal guidance laws have been verified in Figure 2
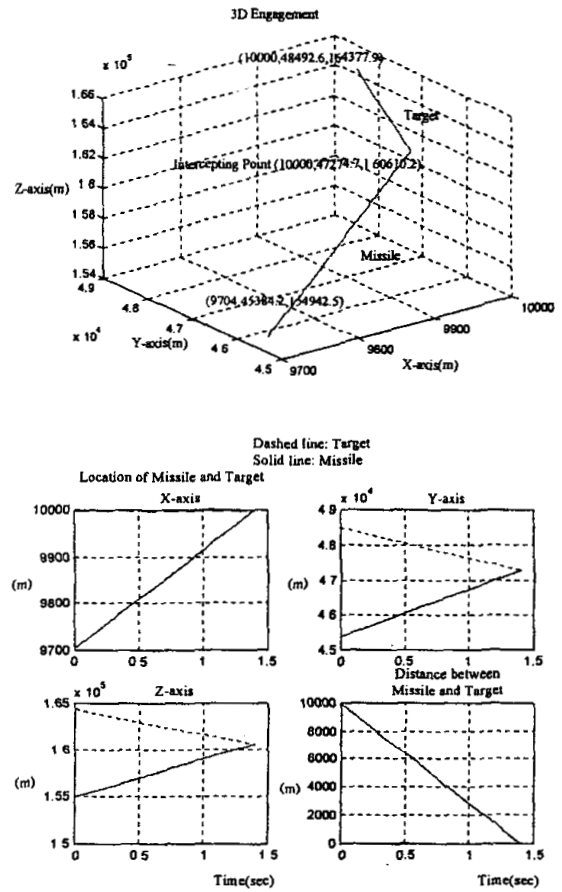

Figure 2: Simulation Results

\section{Conclusions}

The overall process of intercepting a ballistic missile includes two parts: the midcourse phase and the terminal phase. In this paper, we focus on the midcourse phase of the interception, which is a period of time lasted until the missile is close enough to the target such that the sensor located on the missile can lock on the target. Considering the properties of the TVC and the non-ideal conditions during the midcourse phase, we employ the controller integrated by the optimal guidance law, where the time-to-go of the missile is unnecessary to estimated, and the sliding mode autopilot system, which can adjust the attitude of the missile even when considering the uncertainty 
of the inertia of the missile and the aerodynamic force exerted on the missile.

Various simulations have been adopted to verify the feasibility of the integrated midcourse guidance system. Simulations base on the terminal guidance law by $\mathrm{Fu}[3]$ have been adopted, too.

\section{Bibliography}

[1] Kuang-Yow Lian and Li-Chen Fu, "Nonlinear Autopilot and Guidance for a Highly Maneuverable Missile," Proc. Amercian Control Conference, pp.2293-2297,1994.

[2] Li-Chen Fu and Wei-Der Chang, "A Nonlinear Constant Bearing Guidance and Adaptive Autopilot Design for BTT Missiles," Proc. Amercian Control Conference, pp.2774-2778,1997.

[3] Li-Chen Fu, Chi-Wang Tsai and Fu-Kuang Yeh, "A Nonlinear Missile Guidance Controller with Pulse Type Input Devices," Proc. Amercian Control Conference, pp.3753-3757,1999.

[4] Ha, I., and Chong, S., "Design of a CLOS Guidance Law via Feedback Linearization," IEEE Trans. on Aerospace and Electronic Systems, vol. 28, no. 1, pp. 51-63, 1992.

[5] Jie Huang and Ching-Fang Lin, "A Modified CLOS Guidance Law Via Right Inversion," IEEE Trans. on Aerospace and Electronic Systems, vol. 31, no. 1, pp. 491-495, 1995.

[6] Frank L. Lewis and Vassilis L. Symos, Optimal Control, John Wiley \& Sons, Inc., 1995.

[7] Slotine, J.J.E. and W. Li, Applied Nonlinear Control, Prentice-Hall, 1991.

[8] I. Rusnak and L. Meir, "Optimal Guidance for Acceleration Constrained Missile and Maneuvering Target," IEEE Trans. on Aerospace and Electronic Systems, vol. 26, no. 4, pp.618-624, 1990.

[9] Mohammad-Ali Massoumnia, "An Optimal MidCourse Guidance Law for Fixed-Interval Propulsive
Maneuvers," Proc. American Control Conference. San Francisco, CA, USA, Jun 2-4, pp. 43-45, 1993.

[10] Gano B. Chatteji and Minjea Tahk, "A Quatemion Formulation for Boost Phase Attitude estimation, Guidance and Control of Exoatmospheric Interceptors," Proc. American Control Conference, Pittsburgh, PA, USA, Jun 21-23, pp. 1561-1566, 1989.

[11] Ki Baek Kim, Myung-Joon Kim and Wook Hyun Kwon, "Modern Guidance Laws via Receding Horizon Control without the time-to-go," Proc. the $3^{\text {th }}$ IEEE Conf. on Decision \& Control, Tampa, Florida, USA, pp. 4202-4207, 1989.

[12] Bong Wie and Peter M. Barba, "Quatemion Feedback for Spacecraft Large Angle Maneuvers," Journal of Guidance, vol. 8, no. 3, pp. 360-365, 1985.

[13] B. Wie, H. Weiss and A. Arapostathis, "Quaternion Feedback Regulator for Spacecraft Eigenaxis Rotations," Journal of Guidance, vol. 12, no. 3, pp. 375-380, 1989.

[14] Kuang-Yow Lian, Li-Sheng Wang and Li-Chen $\mathrm{Fu}$, "Globally Valid Adaptive Controllers of Mechanical Systems," IEEE Trans. of Automatic Control, vol. 42, no. 8, pp. 1149-1154, 1997.

[15] Yon-Ping Chen and Shih-Che Lo, "Sliding-Mode Controller Design for Spacecraft Attitude Tracking Maneuvers," IEEE Trans. on Aerospace and Electronic Systems, vol. 29, no. 4, pp.1328-1333, 1993.

[16] J. -J. E. Slotine and M. D. Di Benedetto, "Hamiltonian Adaptive Control of Spacecraft," IEEE Trans. on Automatic Control, vol. 35, no. 7 , pp.848-852, 1990.

[17]C. F. Lin, "Analytical Solution of Optimal Trajectory-Shaping Guidance," Journal of Guidance, vol. 10, no. 1, pp. 61-66, 1987. 